\title{
Investigation of failure mode interaction in laminated composites subjected to compressive loading
}

\author{
Pavana Prabhakar, Wei H Ng†, Anthony M Waasªnd Ravi Raveendra ${ }^{\ddagger}$
}

\begin{abstract}
Failure mode interactions when a laminated fiber reinforced composite is subjected to compression is studied here. Experimental results of two different type of fiber-reinforced laminated composites subjected to compression are obtained. Results from a 8-layer microlaminate for predicting the compression response and failure are discussed. A simple 3 layer model is adopted to investigate the interaction between kinking and delamination. To account for delamination, cohesive elements are used between laminae. The competition and interaction between delamination failure modeled through cohesive elements, and fiber kinking captured through initial geometric imperfections and matrix non-linearity are studied in detail.
\end{abstract}

\section{Introduction}

Fiber reinforced composite materials when subjected to compression may exhibit different types of failure mechanisms, namely, microbuckling leading to kinking, delamination, and matrix damage. These different modes of failure occur either separately or simultaneously depending on the loading which affects the global response of the laminate. Therefore, it is of importance to study the interaction between these different types of failure mechanisms. In order to predict and investigate these mechanisms, micro-laminate models approximately representing the actual laminate are chosen for study. The computational modeling is aided by compression tests conducted on two different types of laminates in order to obtain the global stress-strain response along with failure modes. A comparison between results obtained from the micro-laminate models and experiments is studied here. The computational models incorporate matrix inelasticity, initial geometric imperfections and delamination failure. The interaction between the different failure mechanisms are studied in detail.

The prediction of damage initiation in compressively loaded angle-ply laminates and notched laminates subjected to uniaxial and biaxial planar loads at room and elevated temperature has been previously studied by Ahn and Waas. ${ }^{1,2}$ In the former, damage initiation as a function of ply angle is studied in detail while in the latter a local-global model is presented to capture failure by kinking starting at the notch. The review paper by Schultheisz and Waas ${ }^{3}$ summarizes past work related to characterizing compression strength, and a discussion is provided on how the compressive strength is affected by different mechanisms such as microbuckling, kinking, fiber failure and longitudinal cracking (or splitting). Past studies and theories have focussed on the effect of a single failure mechanism on the compressive strength of composites, whereas it is recognized that in multi-directional laminates, initiation and spreading of failure occurs through several competing and interacting failure mechanisms. Yerramalli and Waas, ${ }^{4}$ Basu et al. ${ }^{5}$ and Jumahat et al. ${ }^{6}$ have introduced failure models for composites subjected to combined loads, including compression. These models predict the compressive strength of a unidirectional composite subjected to compressive loading, in fairly good agreement with the experimentally measured values. Extensions of these ideas to multi-directional laminates is lacking. The aim of this paper is to formulate a methodology to predict the response and failure of a multi-directional laminate when subjected to compression, taking into account interaction between different

\footnotetext{
*Graduate Student, Department of Aerospace Engineering, 1320 Beal Avenue, Ann Arbor, MI 48109-2140, and AIAA Student Member.

${ }^{\dagger}$ Post-Doctoral Researcher, Department of Aerospace Engineering, 1320 Beal Avenue, Ann Arbor, MI 48109-2140

$\ddagger$ Felix Pawlowski Professor of Aerospace Engineering, Department of Aerospace Engineering, 1320 Beal Avenue, Ann Arbor, MI 48109-2140, and Fellow, AIAA.

${ }^{\S}$ Comet Technology Corporation, Ann Arbor, Michigan
} 
failure mechanisms. The focus of this paper is on the competition between fiber microbuckling/kinking and delamination failure.

\section{Experiments}

Compression tests were conducted on fiber-reinforced composite laminates to understand the mechanisms of compressive failure and to examine the influence of stacking sequence on these mechanisms, and lamina thickness. The properties of the matrix and the fiber are held fixed. The influence of two important types of failure mechanisms, namely delamination and kinking and their interaction on the compression strength was the main focus of the study. In the following sections, details about the experimental set-up, stacking sequence (also referred to as layups) of different specimens and the results of compression tests are given.

\section{A. Test Fixture}

The Wyoming Combined Loading Compression (WCLC) test fixture shown in Fig. 1 was used to carry out the compression tests in association with a MTS loading frame. Specimens in the form of laminated strips with nominal dimensions of 0.5 inch x 5.25 inch x " $t$ " inches, where, " $t$ " is variable, are sandwiched between the large metal blocks of the fixture and the ends of the fixture are compressed between the flat loading platens of a MTS testing frame as shown in Fig. 1 and Fig. 2. The blocks act as anti-buckling guides during the tests, supporting a large portion of the specimen length, and providing a length of 0.25 inch as the gage length. This results in the measured compressive strength to be as close as possible to the actual compressive strength of the material, uncontaminated by any tendency towards flexural buckling.

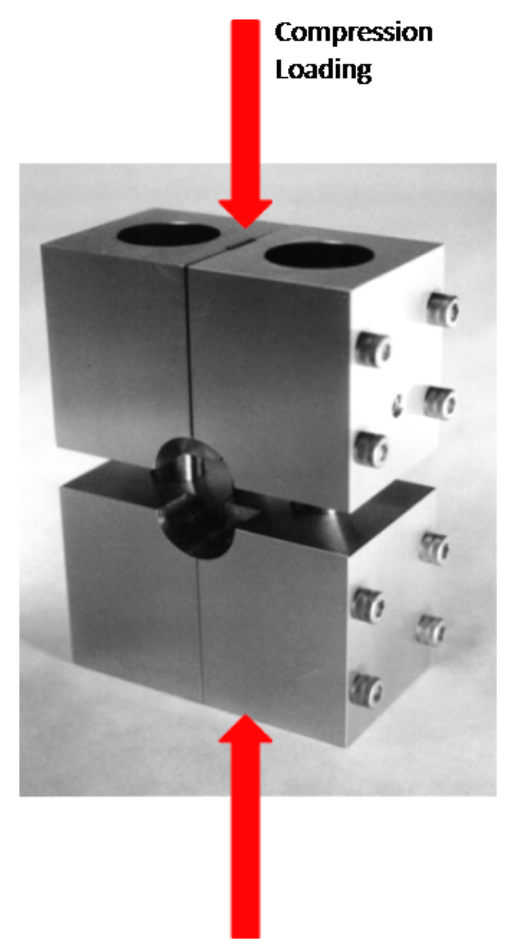

Figure 1. Assembled fixture with specimen installed

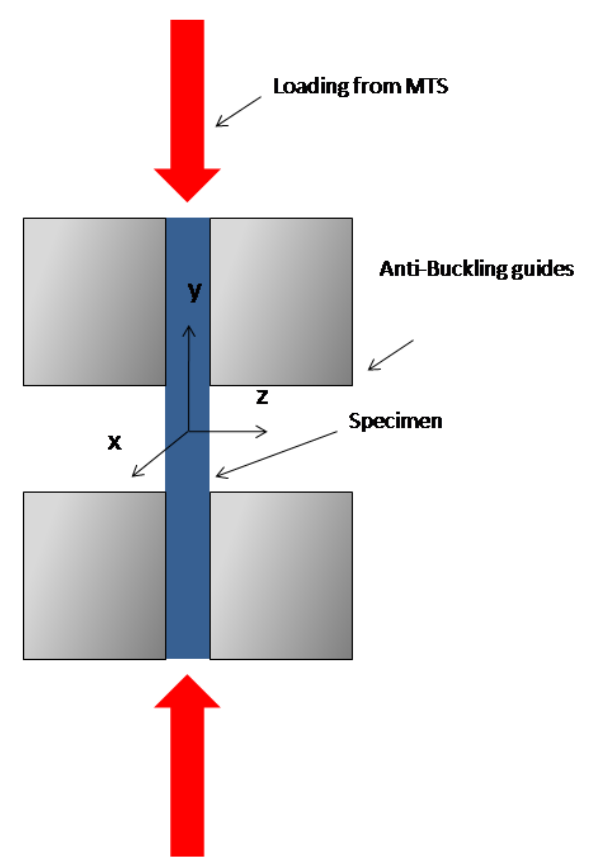

Figure 2. Sketch of loading configuration

\section{B. Specimens}

Specimens of four different layups and of varying thickness were tested under compression. Three of the four specimens are also used to study the effects of scaling the thickness of each lamina on the compressive strength of the laminates. As indicated in Table 1, Type A laminates of Layups 1, 2 and 3 have the same orientation in the layups, but the thicknesses of the layups are scaled up by stacking multiple layers of the 
same orientation. The Type $\mathrm{C}$ laminate has a completely different stacking sequence, however, because it contains the same basic block of lamina groups, $(-45 /+45 / 90 / 0)$, the four different laminates shown in Table 1 have the same in-plane stiffnesses as will be shown later. All the specimens are of size 0.5 inch x 5.25 inch which results in a gage length of 0.25 inch when placed in the WCLC fixture as shown in Fig. 2.

Table 1. Types of Laminates

\begin{tabular}{cccc}
\hline \hline Type of Laminates & $\mathrm{L}$ (in) & $\mathrm{W}$ (in) & $\mathrm{t}$ (in) \\
\hline Type A, Layup 1: $[-45 /+45 / 90 / 0] \mathrm{s}(8$ plies $)$ & 0.25 & 0.5 & 0.049 \\
Type A, Layup 2: $[-452 /+452 / 902 / 02] \mathrm{s}(16$ plies) & 0.25 & 0.5 & 0.086 \\
Type A, Layup 3: $[-454 /+454 / 904 / 04] \mathrm{s}(32$ plies $)$ & 0.25 & 0.5 & 0.176 \\
Type C, Layup 1: $[(-45 /+45 / 90 / 0) 6] \mathrm{s}(48$ plies $)$ & 0.25 & 0.5 & 0.25 \\
\hline
\end{tabular}

\section{Zero Laminae}

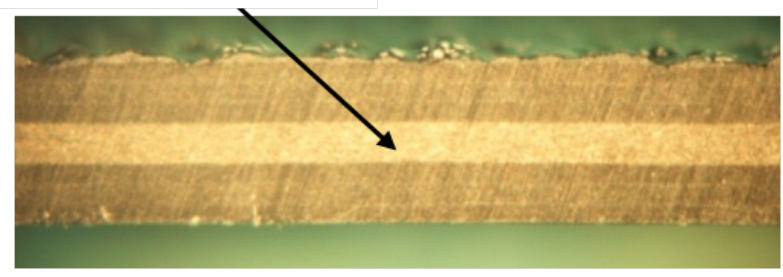

Figure 3. Type A specimen : 8 ply specimen prior to testing

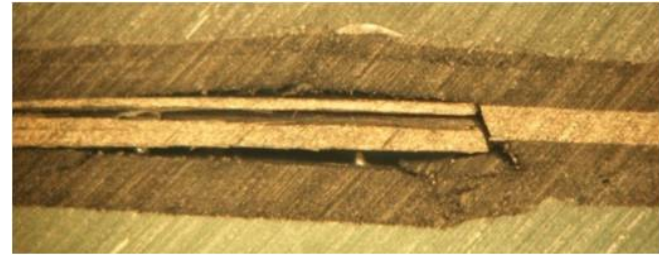

Figure 4. Type A specimen : 8 ply specimen after failure

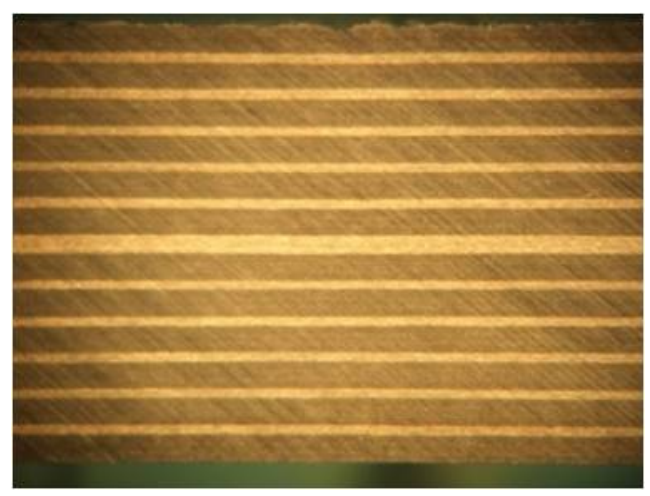

Figure 5. Type C specimen : 48 ply specimen prior to testing

\section{Kink Band}

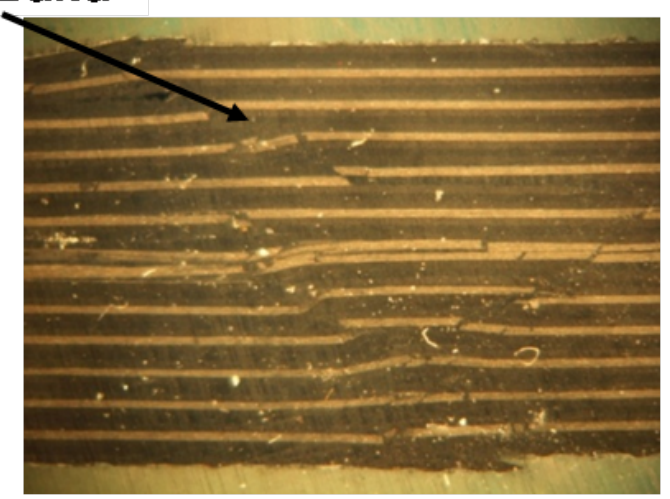

Figure 6. Type C specimen : 48 ply specimen after failure

Typical images of the laminates mentioned above are shown in the Fig. 3,Fig. 4,Fig. 5 and Fig. 6. Fig. 3 and Fig. 5 display the images of Type A (Layup 1) and Type C (Layup 1) laminates in the pristine configuration, and Fig. 4 and Fig. 6 display the images of the failed specimens of the corresponding laminates. We observe that the failed Type A specimen shows delamination occurring at the interface of the laminae. Whereas, the failed Type $\mathrm{C}$ specimen shows distinct kink bands in the zero degree laminae formed through the thickness of the specimen. As will be discussed later, the strain fields that persist upto and beyond failure indicate that there is fairly uniform deformation corresponding to an initial linear relation between applied load and strain. At a critical value of the applied end displacement, a sudden transition in the stability of the specimen occurs and this leads to catastrophic failure with a significant reduction in the measured load. 


\section{Details of the Tests}

The compression tests are carried out at an external displacement control loading rate of $0.0004 \mathrm{in} / \mathrm{sec}$ in a MTS hydraulic test frame. The "macroscopic" stress is calculated as the total load obtained from a load cell that is placed in-line with the specimen, divided by the initial undeformed cross sectional area of the specimen. The "macroscopic" strain is usually determined using the end displacement measured from the axial movement of the ends of specimen, but, in the present study, strains in the gage section of the specimen are measured using digital image correlation (DIC). These strain fields are then analyzed to extract an "averaged" macroscopic strain that is used for presenting the results. The ARAMIS, commercially available software package is used to perform the DIC analysis. ARAMIS is a non-contact and material independent displacement measuring system which gives displacements, strains and velocities as a function of time. The side surface of the specimen (through the thickness) which is to be imaged has a speckle pattern with random black dots over a white background, created using an air-brush. The side surface that is imaged is the surface with a normal in the $\mathrm{z}$ - direction, where the axes are as indicated in Fig. 2. A series of images are taken during the experiment, and these images are post-processed by ARAMIS to calculate the displacements and strains.

Using ARAMIS, the strains $\epsilon_{x x}, \epsilon_{y y}$ and $\epsilon_{x y}$ are computed as follows. The field of view of the side surface measures 0.049 inch $\times 0.25$ inch, 0.086 inch $\times 0.25$ inch, 0.176 inch $\times 0.25$ inch and 0.25 inch $\times 0.25$ inch for the Type A specimens of thickness 0.049 inch, 0.086 inch, 0.176 inch respectively and for the Type C specimen of thickness 0.25 inch. An area in the center of this image that corresponds to a height of 0.25 inch and which spans the specimen thickness, is used as the area over which the strain $\epsilon_{y y}$ in the loading direction is averaged. This strain is referred to as the macroscopic strain and is used as the horizontal axis, while the vertical axis represents macroscopic stress in the global stress-strain response of the laminate subjected to compression.

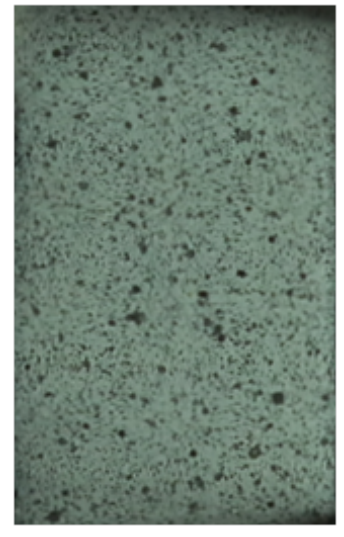

Figure 7. Unloaded

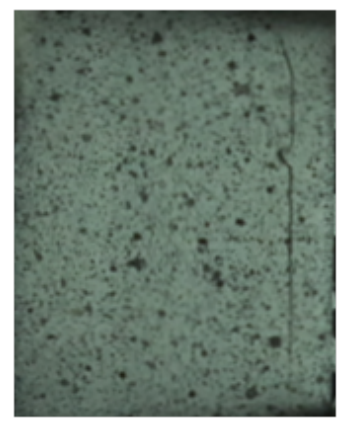

Figure 8. Peak load

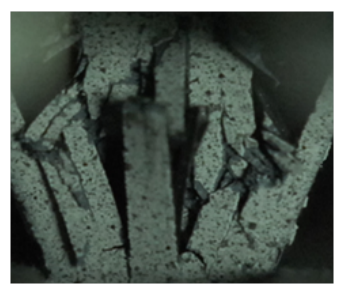

Figure 9. Failed

Fig. 7, Fig. 8 and Fig. 9 show a series of images of a Type A (Layup 3) specimen as a function of macroscopic stress state. The first image, a reference image that is used for the DIC calculations, corresponds to the unloaded state while the next two images correspond to an instance near the peak load and immediately thereafter. The images and the corresponding locations on the stress-strain plot are marked as A, B and C. In image B, a delamination crack is already visible and is identified as the first event that may trigger the catastrophic failure which, as seen in image $\mathrm{C}$, also induces kinking in the zero lamina in the post-peak regime. This type of failure mechanism that initiates the catastrophic failure was visible in all of the Type A laminates, regardless of the thickness scaling, and is also further supported by the DIC strain field analysis which will be discussed in the next section.

\section{STRAIN ANALYSIS OF LAMINATES USING DIC - ARAMIS}

The DIC images of the cross-sectional area of a typical Type A (Layup 3) specimen are shown in Fig. 10a, Fig. 10b and Fig. 10c. The specimen is loaded in the global y-direction. Fig. 10a, b and c display the strain distribution on the cross-sectional area along the global x-direction. We notice that the distribution is banded along the thickness. This is due to the different layers present in the specimens. We also observe that as 
the loading is increased, the positive strain between the layers +45 and -45 increases tremendously, and the specimen delaminates at that interface as clearly shown in Fig. 10c. To corroborate the above statement, the strain distributions $\epsilon_{x x}$ and $\epsilon_{x y}$ along a line on the cross-section are also plotted. It is clear from Fig. 11b and Fig. 11b that as the load is increased, the transverse strain $\left(\epsilon_{x x}\right)$ and the shear strain $\left(\epsilon_{x y}\right)$ attain maximum values at the interface between +45 and -45 layers. Upon further loading, the transverse and shear strains tend to infinity as the specimen delaminates at the interface on the right (refer to Fig. 11c and Fig. 12c). In contrast, such strain concentrations immediately prior to failure are absent in the lay-up C specimens. In these specimens, as seen in Fig. 13a,b and c the strain localizes within the zero-plies in the interior of the specimen and away from the edges. Thus, in Type $\mathrm{C}$ specimens, the strains are largest in the zero-ply and not at the interfaces. This finding corresponds to the kink banding (without delamination) that is responsible for initiating failure in type $\mathrm{C}$ specimens. In summary, there is a distinction between the mechanisms of failure in Type A and Type $\mathrm{C}$ specimens. Type A specimens appear to initiate failure by delamination followed by kink banding occurring simultaneously or in the post-peak regime. Whereas, in Type $\mathrm{C}$ specimens, the transverse and shear strains across interfaces is not critical. Instead, a strain concentration occurs within the interior zero laminae, which in the post-experiment images, appear as kinked.
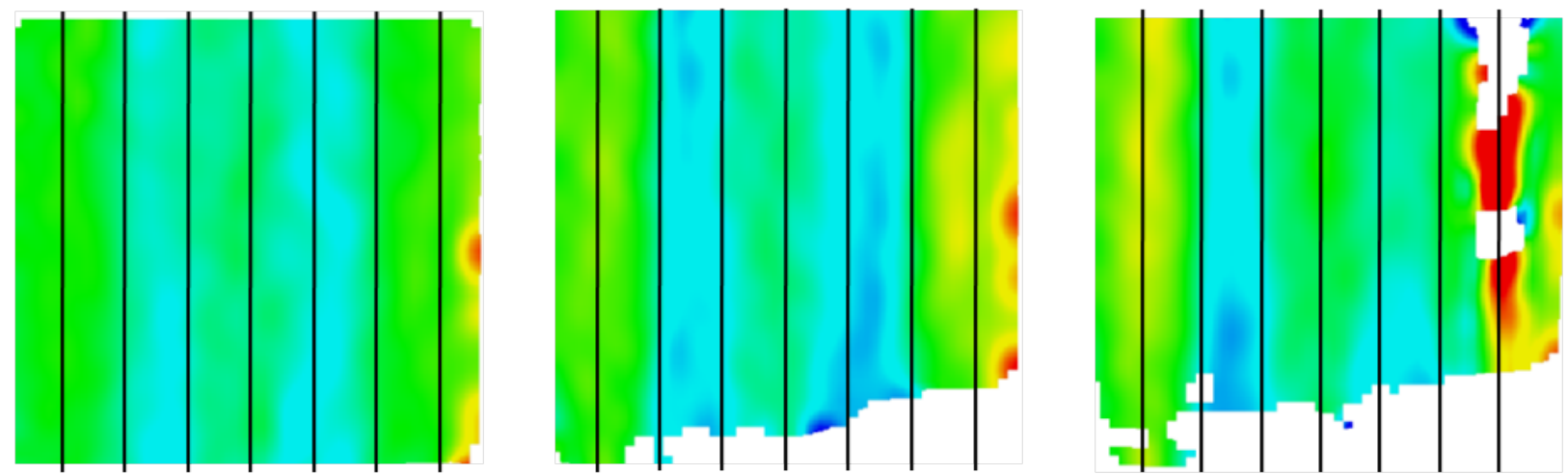

Figure 10. Transverse strain distribution on the side surface for Type-A 16-ply laminate
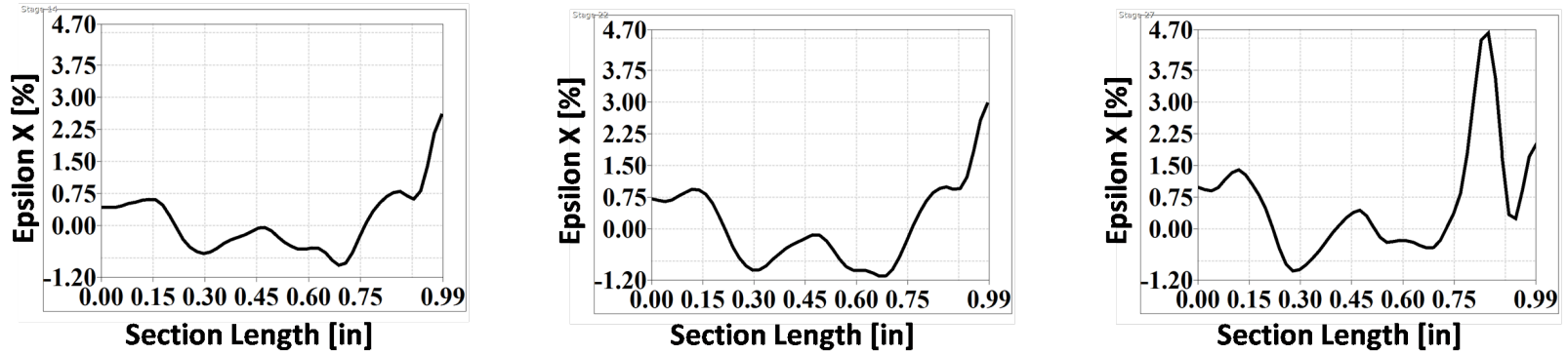

Figure 11. Transverse strain distribution across the side surface for Type-A 16-ply laminate
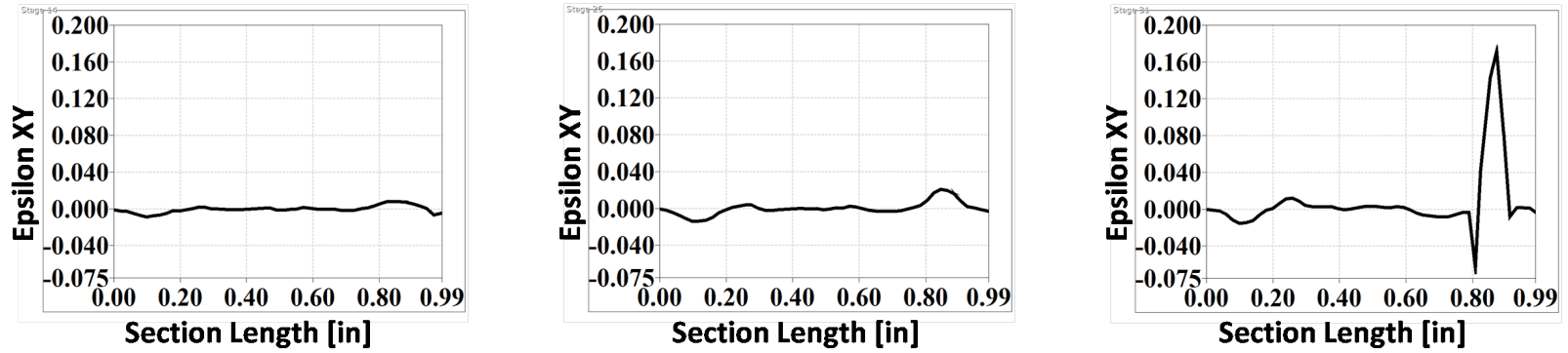

Figure 12. Shear strain distribution across the side surface 

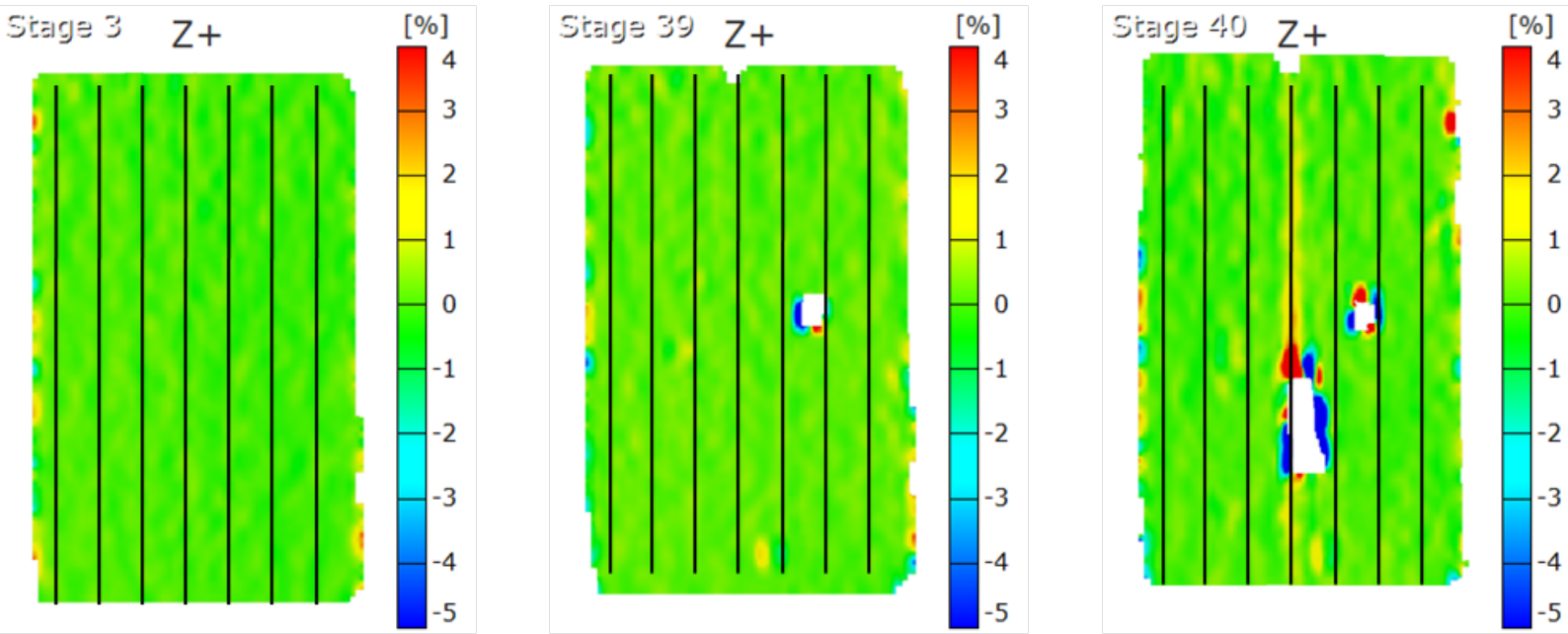

Figure 13. Transverse strain distribution on the side surface for Type-C 48-ply laminate
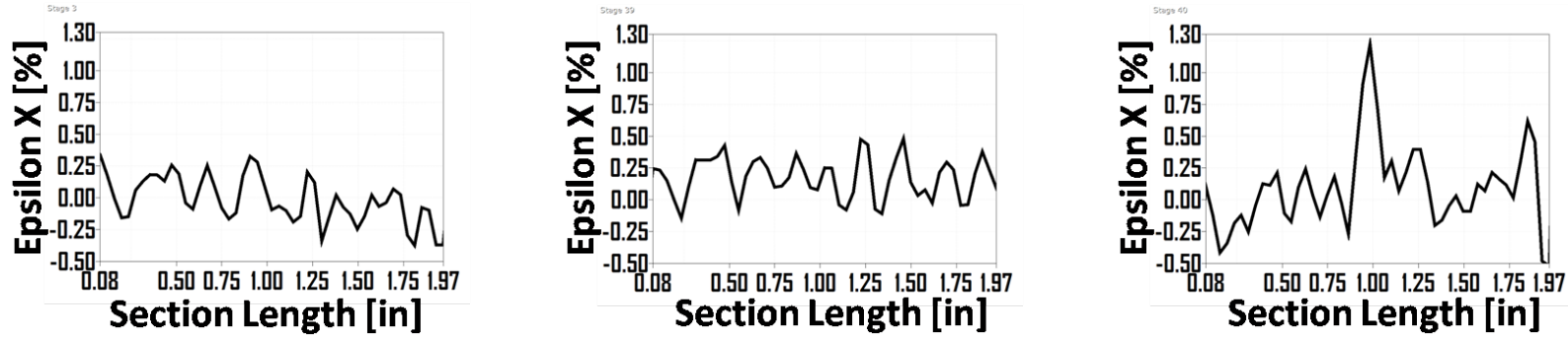

Figure 14. Transverse strain distribution across the side surface for Type-C 48-ply laminate
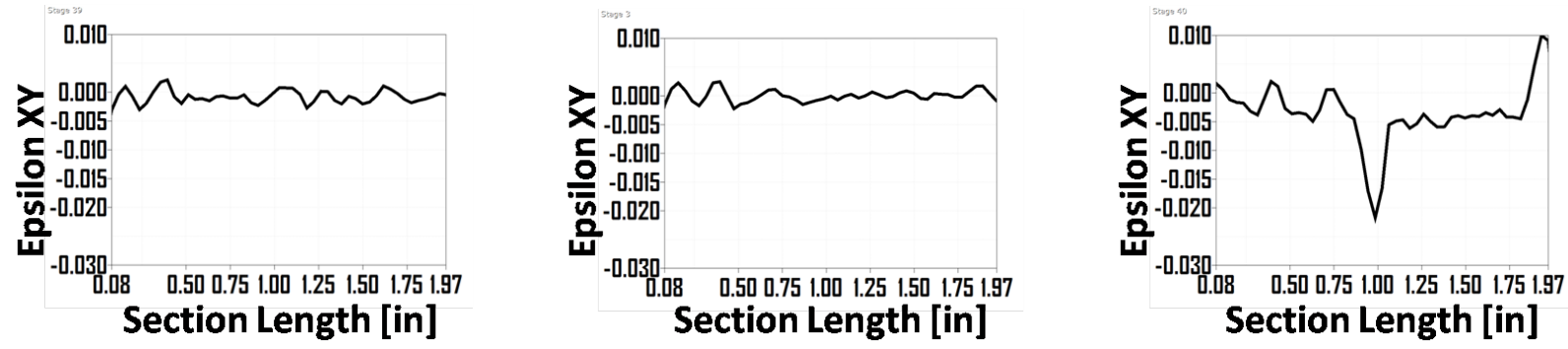

Figure 15. Shear strain distribution across the side surface for Type-C 48-ply laminate

\section{E. GLOBAL STRESS - STRAIN CURVES}

The "macroscopic" stress - strain curves are plotted for the three Type A specimens, and are shown in Fig. 16. Here, the macroscopic strain is the average strain within the DIC imaged area as explained earlier. We notice that for all the three layups of Type A specimens, the initial stiffness is almost equal and is in the range $6015 \mathrm{GPa}$. This initial stiffness is the tangent modulus of the responses plotted in Fig. 16, evaluated at a macroscopic stress of about $150 \mathrm{MPa}$. Also, the peak stress/compressive strength of all the specimens are approximately in the range 450 - $600 \mathrm{MPa}$. Therefore, we can conclude that the scaling of the thickness of laminae has no effect on the "macroscopic" compression strength of the composite. 


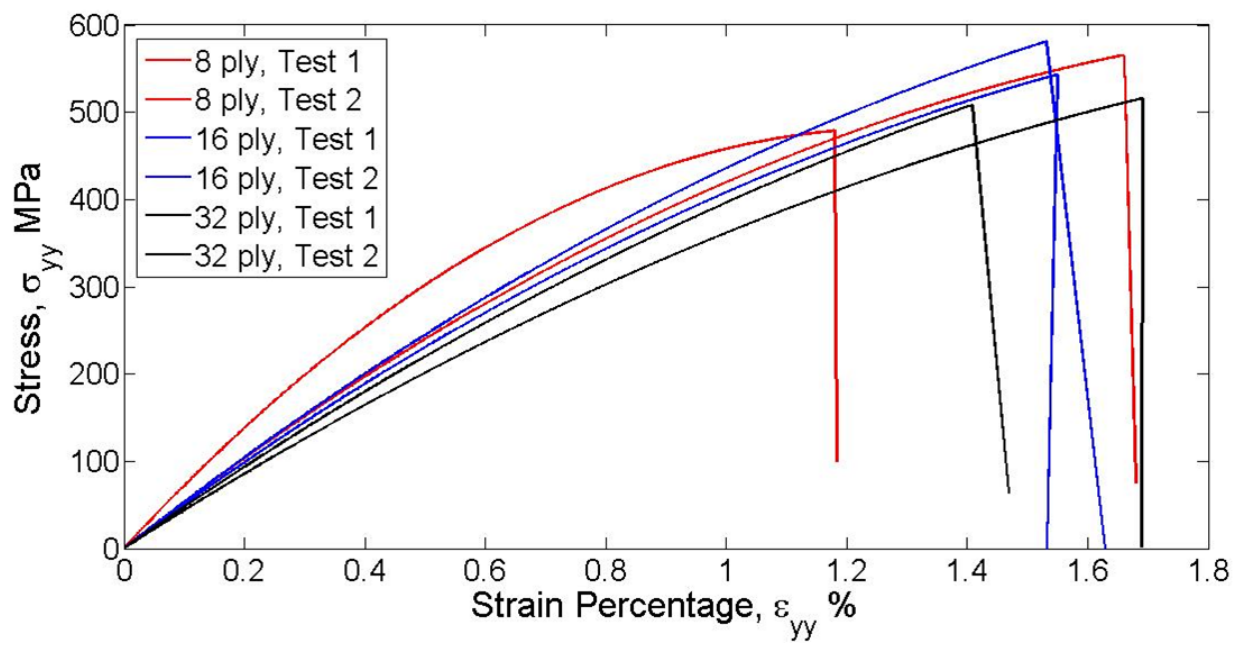

Figure 16. Experimental "macroscopic" stress - "macroscopic" strain curves

\section{Computational Modeling}

In order to explain the different and possibly interactive failure mechanisms that were observed for the different types of laminates, computational models based on micromechanics were developed and executed using the finite element method in conjunction with the commercial software ABAQUS. While the micromechanics provides a way to capture individual fiber-fiber interactions and fiber-matrix interactions, it is neither convenient nor expedient to model every fiber in every lamina. Instead, the intent here is to capture the transversely isotropic nature of the laminae, the fact that there is a thin matrix layer between laminae as shown in the images of Fig. 3 earlier, and the fact that there is a certain pattern to the stacking sequences in the Type A and Type C laminates. Outline of the procedure to capture these features is outlined next.

Two models are developed based on micromechanics and the ease of modeling. The first model is an 8-layered model with a layup of $[-45 /+45 / 90 / 0 / 0 / 90 /+45 /-45]$, and comprising of discrete fibers and matrix. The individual layers are scaled down to orders of 3 times the fiber diameter, and hence the model is named as a "micro-laminate". The second model consists of a representative micro-section of a three layered composite laminate with a [90/0/90] layup and and is currently used to investigate the interaction between kinking and delamination modes of failure. In both the models, the fibers are assumed to be orthotropic and linearly elastic material. The matrix is taken to be isotropic, with response extending beyond the linear elastic regime. The plot of equivalent stress-equivalent strain for in-situ matrix used is shown in Fig. 17. The properties of fiber are given in Table 2 .

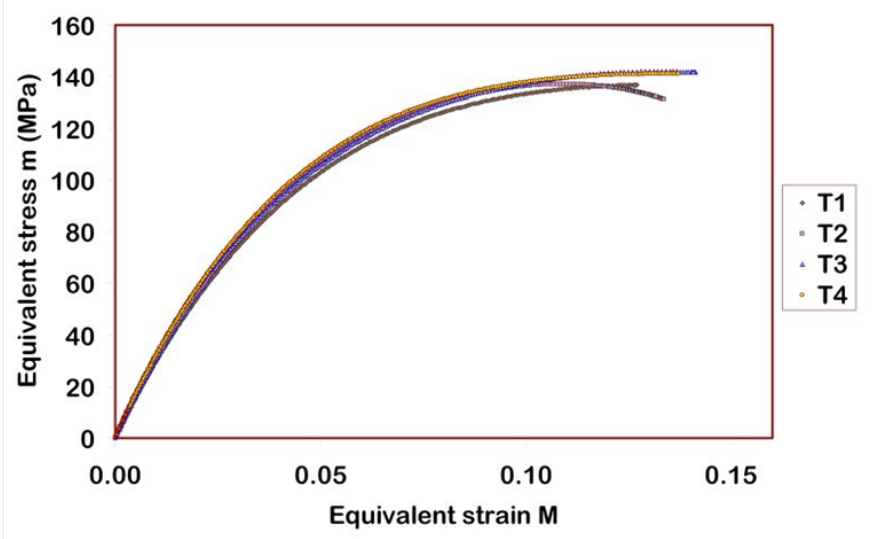

Figure 17. Equivalent stress-equivalent strain for in-situ matrix 
Table 2. Fiber Properties

\begin{tabular}{cccccccccc}
\hline \hline & $E_{11}(\mathrm{GPa})$ & $E_{22}(\mathrm{GPa})$ & $E_{33}(\mathrm{GPa})$ & $G_{12}(\mathrm{GPa})$ & $G_{13}(\mathrm{GPa})$ & $G_{23}(\mathrm{GPa})$ & $\nu_{12}$ & $\nu_{13}$ & $\nu_{23}$ \\
\hline Fiber & 310 & 20.5 & 20.5 & 32.8 & 32.8 & 7.6 & 0.28 & 0.28 & 0.28 \\
\hline
\end{tabular}

\section{A. 8-LAYER SCALE MODEL SIMPLIFICATION OF THE LAMINATE}

An 8-layered finite element model is created with a layup of $[-45 /+45 / 90 / 0 / 0 / 90 /+45 /-45]$. This 8 layer stack is a repeat unit that is representative of the Type A laminates. It is also approximately representative of the Type $\mathrm{C}$ laminates (for the Type $\mathrm{C}$ laminates, this lay-up is identical to the 8 laminae at the center of the laminate - see Table 1). Each lamina is a hexagonally packed transversely isotropic layer having three rows of fibers. The fiber diameter and volume fraction are captured as measured from the laminates, consequently these laminae are scaled laminae that capture these two features but do not have the same thickness as the laminae in the tested laminates. Fig. 18 shows the scale model simplification of the laminates. In this figure, both, a 3D version and a 2D version are shown. Calculations with the $3 \mathrm{D}$ version are not reported here, instead results are generated using the $2 \mathrm{D}$ plane-strain model only.

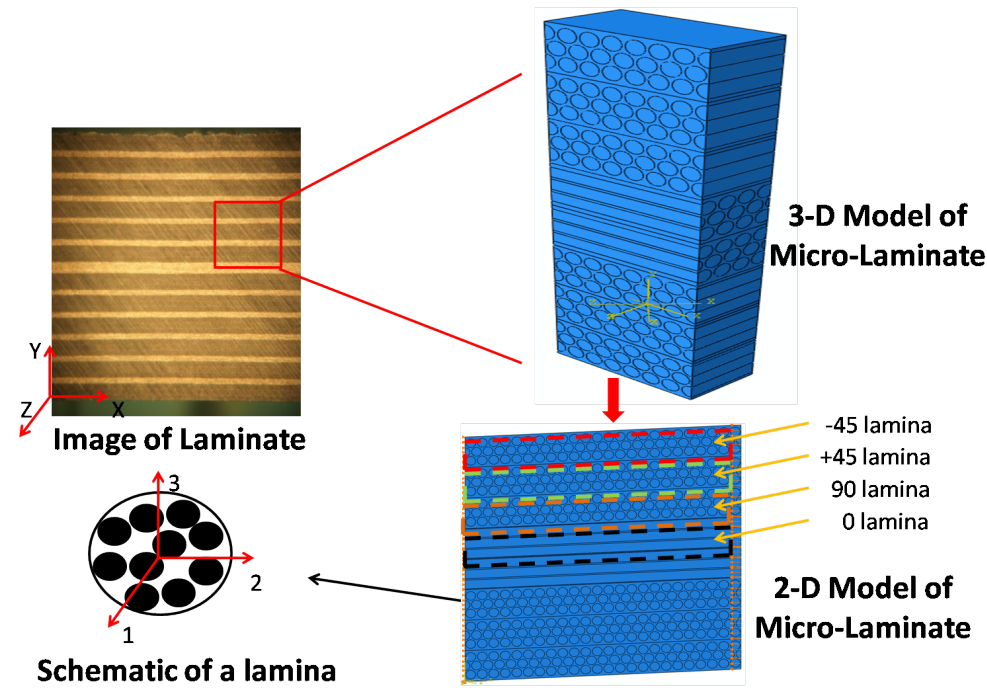

Figure 18. 8-layer scale model simplification of the laminate
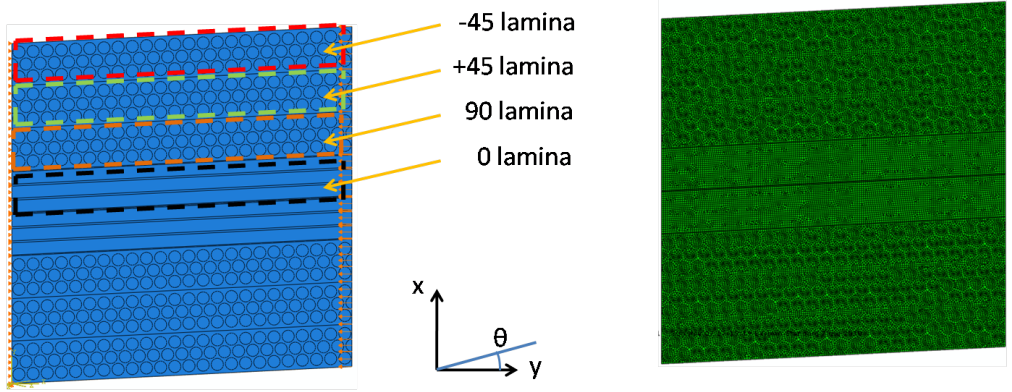

Figure 19. 2-D 8-layer model of the laminate

In this 2D model, shown in Fig. 19, circular cross-section fibers are used in the +45 and -45 laminae instead of the elliptical cross-section fibers, as seen in the XY plane. Because of the use of these circular crosssection fibers, the fiber properties in these laminae are adjusted so that they correspond to the properties of the +45 and -45 laminae in the XY-plane. This model represents the Type $\mathrm{C}$ and Type A (Layup 1) very closely. In order to model the Type A-Layup 2 and Layup 3 laminates, the thickness of each layer of the 
model can be doubled or tripled, respectively.

\section{OVERVIEW OF THE MODEL}

The overall dimension of the 2-D model is $282 \mu m \times 188 \mu m \times 0.001 \mu m$. The fiber radius is $3 \mu m$. The model is meshed with 2-D bilinear quadrilateral elements in ABAQUS given by CPE4, which is a plane strain approximation of the 3-D case. A slight imperfection is imparted to the model to account for fiber misalignment. Previous work ${ }^{8}$ has shown that initial misalignment angles of 0.5 to 2 degrees of the zero laminae bound the distribution of fiber misalignment that is typical of carbon fiber reinforced pre-preg aerospace laminates. An exaggerated view of the misaligned microlaminate model is shown in Fig. 19. The unloaded state is stress free. The edge $\mathrm{AB}$ of the model is prevented from motion in the y-direction (refer to Figure 15), and the corner $\mathrm{A}$ is fixed against movement in the global $\mathrm{x}$ and $\mathrm{y}$ directions. The edge CD is subjected to compression along the negative y-direction in a displacement control manner. The Riks method option available in ABAQUS v6.10, which is an arc-length solution scheme, is adopted to conduct the response analysis. As shown in previous studies $\left({ }^{?}\right)$, this method captures unstable equilibrium paths (path in the load vs. loaded edge displacement graph that show snap-back response) that can occur at limit points, as will be discussed later.

\section{B. GLOBAL STRESS-STRAIN CURVE}

\section{Imperfection Sensitivity Analysis}

The model is seeded with fiber misalignment angles of 0.5 degree, 1 degree and 2 degree, and subjected to compression loading. The macroscopic stress-strain curves for each case are plotted in Fig. 20. Here, macroscopic stress is defined as the total resultant Y-direction reaction force on the edge AB divided by the product of the length $\mathrm{AB}$ and a unit length in the Z-direction, while the macroscopic strain is defined as the total contraction (change in length between the edges $\mathrm{AB}$ and $\mathrm{CD}$ ) divided by the initial length $\mathrm{AD}$.

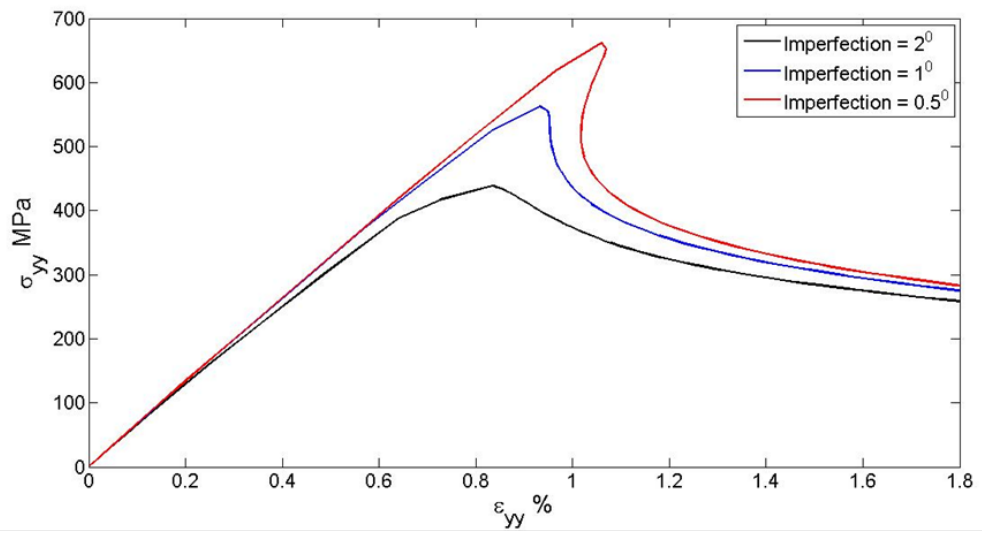

Figure 20. Global stress-strain curve for a range of misalignments

Initially, the microlaminate response is linear until a maximum (limit stress) is reached at which a snapback in the response is observed. As the misalignment increases, this snap back becomes more gradual and the peak stress also diminishes. This $\sigma-\epsilon$ plot is reminiscent of the response of an axially loaded cylindrical shell ${ }^{9}$ and thus, it is of interest to plot the peak stress as a function of imperfection magnitude. Calculations are currently being performed to populate such a plot that would be of great utility for design purposes. The peak stress for misalignment range of 0.50-20 (practical range of misalignment of the zero layers in laminates) is in a range of 400-600 MPa. The initial (linear) stiffness of the response shown in Fig. 20 is approximately $60 \pm 3 \mathrm{GPa}$. These numbers compare very favorably against the measured experimental results reported earlier. 


\section{Strain distribution in the model}

The transverse and shear strain fields are compared side by side at different loading time in Fig. and Fig. . In Fig.'21, (a) corresponds to the initial linear elastic regime of the strain-strain curve, (b) corresponds to the peak load, (c) corresponds to the post-peak regime and (d) corresponds to the plateau load regime. It is noticed that until the peak load is reached, there is almost uniform strain distribution. Fig. 21 (c) and (d), and Fig. 22 (c) and (d) represent $\epsilon_{x x}$ and $\epsilon_{x y}$ in the post peak regime. We notice that there are high strains in the post peak regime at the interface between the different laminae, and also at the fiber - matrix interface in the 00 lamina. The combination of high shear and extensional strains imply that there is scope for delamination at the fiber-matrix interface and between the laminae of the microlaminate.
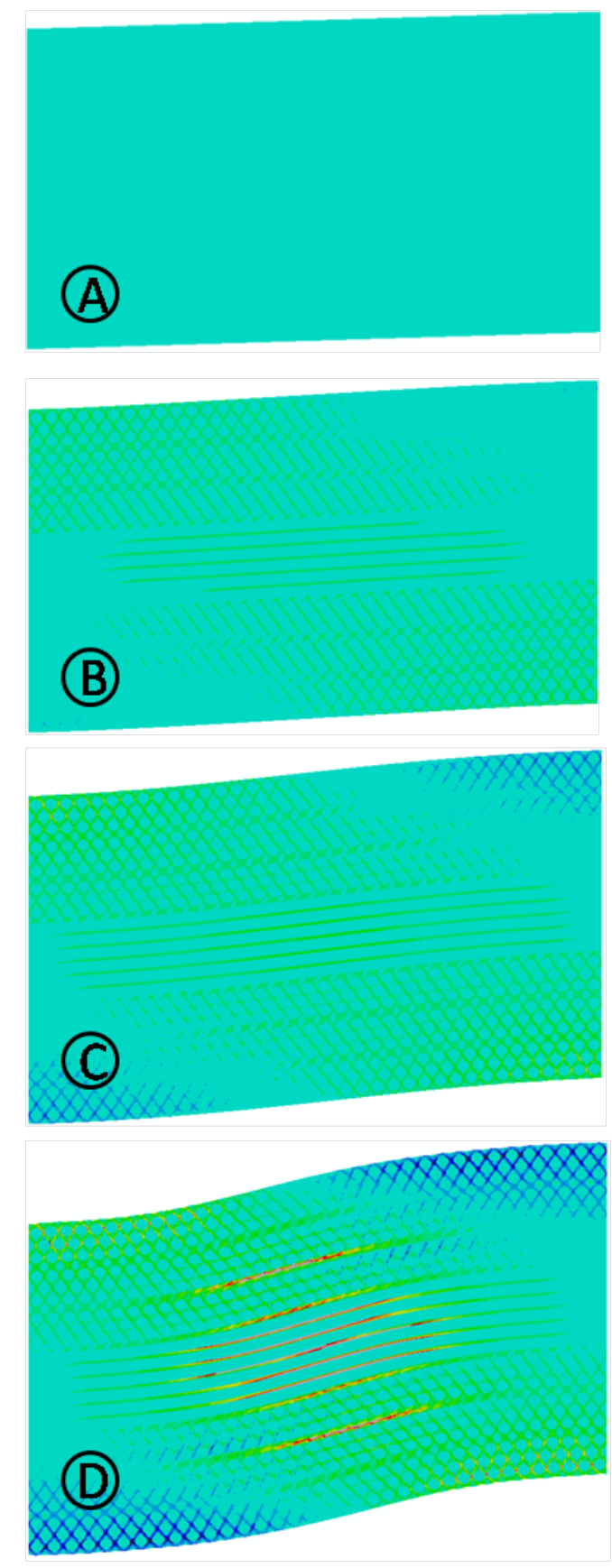

Figure 21. $\epsilon_{x x}$
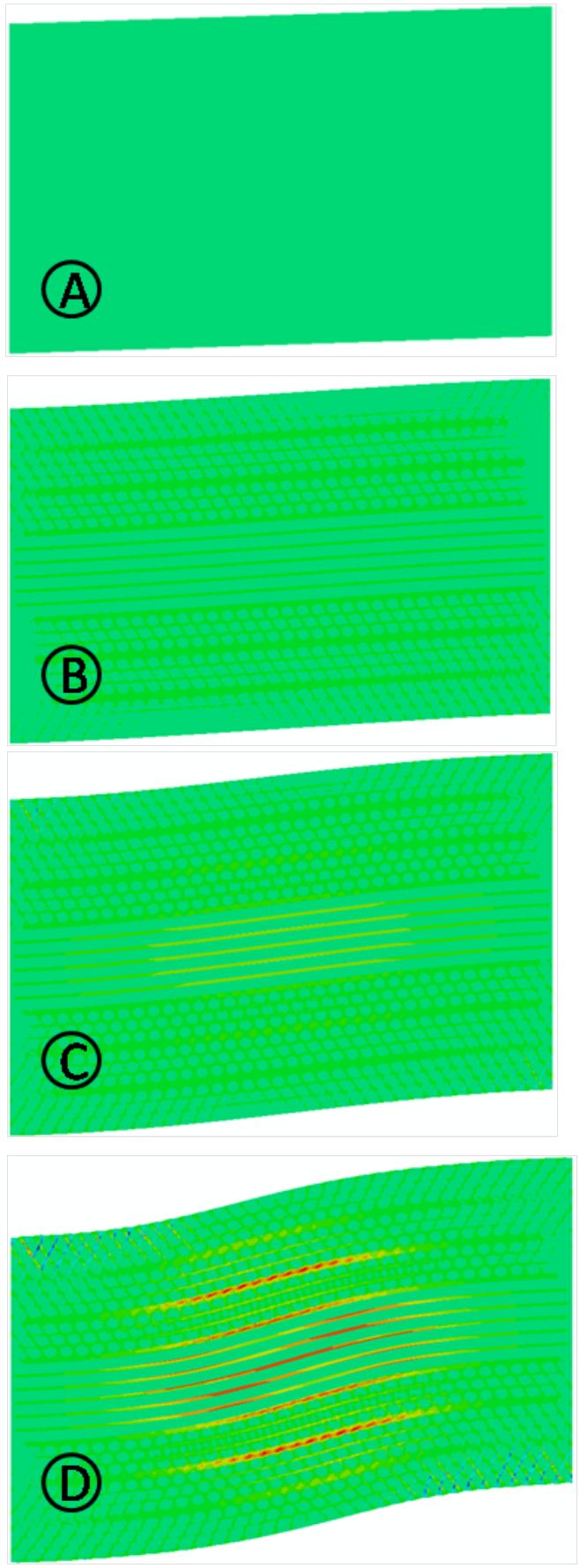

Figure 22. $\gamma_{x y}$ 
The formation of a localized band where the strains are maximum is seen to evolve with continued loading, but the settling of the band, which commences at or just after the peak stress, occurs when the stress is seen to plateau off. Thus, remarkably, this model captures the formation of kink bands as seen in the experimental images that were taken after failure. Therefore, the model currently captures kink banding in the laminates.

\section{COMPARISON OF EXPERIMENTAL RESULTS WITH COMPUTATIONAL RESULTS}

The values of initial stiffness and peak stress, and failure modes observed in all the specimens tested and the numerical model is tabulated in Table 3.

Table 3. Comparison of experimental results with computational results

\begin{tabular}{ccccc}
\hline \hline SPECIMEN or MODEL & INITIAL STIFFNESS(GPa) & PEAK STRESS (MPa) & FAILURE MODE \\
\hline TYPE-A SPECIMENS LAYUP & 1 & $60 \pm 5$ & $559 \pm 39$ & DELAMINATION \\
\hline TYPE-A SPECIMENS LAYUP & 2 & $61 \pm 6$ & $586 \pm 34$ & DELAMINATION \\
\hline TYPE-A SPECIMENS LAYUP & 3 & $59 \pm 8$ & $552 \pm 22$ & DELAMINATION \\
\hline TYPE-C SPECIMENS & $62 \pm 7$ & $640 \pm 40$ & KINKING \\
\hline NUMERICAL MODEL & $63 \pm 2$ & $540 \pm 110$ & KINKING \\
\hline
\end{tabular}

The initial stiffness obtained from the experiments for all laminates and that obtained from the numerical model match pretty well. The peak stresses of Type $\mathrm{C}$ specimens and the numerical model match well along with their failure modes, which is kinking. In order to simulate the delamination failure mode observed in Type A specimens, it is necessary to allow the numerical model to delaminate. This can be achieved by adding discrete cohesive zone model (DCZM) elements at the fiber-matrix interface in the numerical model. A simple 3-layered cross-ply laminate is investigated in the next section as a means to obtain preliminary results.

\section{3-LAYER CROSS-PLY MODEL OF THE LAMINATE}

\section{OVERVIEW OF THE MODEL}

In order to investigate interaction between kinking and delamination failure modes, a simple 2-D 3-layer cross-ply laminate is adopted with the fibers and matrix modeled discretely in each lamina. Following a similar procedure, as explained earlier, the layers are modeled as micro-laminates with only 3 layers of fibers in each lamina but conserving the fiber volume fraction of the laminate. In addition, discrete cohesive zone model (DCZM) elements are added at the interface between the layers which allow the model to delaminate if required. The details of the DCZM elements will be given in the next section. The fibers and the matrix have the same properties as used in the 8-later model earlier. A meshed 3-layer model is shown in Fig. 23.

The model has dimensions of $133.5 \mu \mathrm{m} \times 64.5 \mu \mathrm{m} \times 0.001 \mu \mathrm{m}$, and an initial misalignment of 1 degree is imparted to the model. 2-D bilinear quadrilateral elements are used to mesh the model as before.

\section{DCZM Elements}

The discrete cohesive zone element (DCZM element) adopts a 1D traction law capable of simulating crack formation and propagation, i.e. delamination. The element features the ability to predict delamination initiation based on a traction law that captures the cohesive strength and the fracture toughness, in each fracture mode (mode I and mode II in the current model). The DCZM elements used in this paper have been successfully employed in other studies involving crack propagation as presented in Gustafson and Waas $\left.{ }^{7}\right]$

\section{Discussion on Analysis Techniques}

While buckling analysis is traditionally done using implicit codes with an arc-length method, e.g. RIKs method, the inclusion of delamination in this study introduces convergence difficulties that is beyond the 


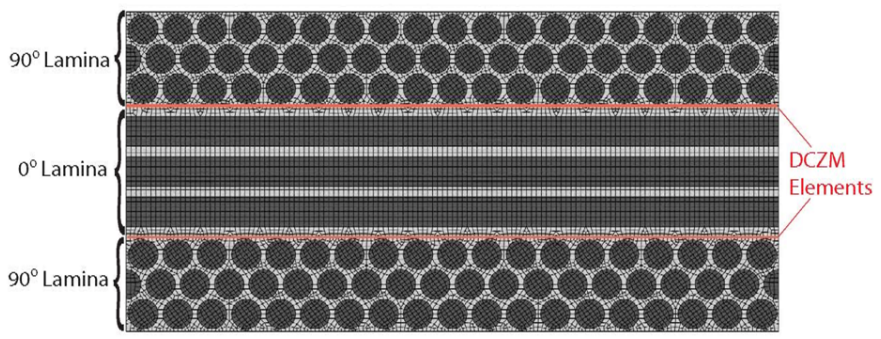

Figure 23. 3-layer cross-ply model of the laminate

capabilities of implicit codes to handle. Even though implicit codes are unconditionally stable, the presence of two unstable failure mechanisms requires very small time steps for convergence, which offsets the unconditionally stable advantage over explicit codes. Therfore, in this study, an explicit code (ABAQUS Explicit $6.10)$ is used to generate the computational results.

One major disadvantage when using explicit code is that it is conditionally stable; time steps need to be below a critical value to ensure a correct solution and the time steps can be much smaller than $1 \mathrm{~s}$. If quasi-loading conditions are modeled exactly, the solution time can be days, which is impractical. Generally, two strategies are employed to obtain a reasonable solution time: The first is to arbitrarily reduce the loading duration, i.e. an actual experiment spanning $100 \mathrm{~s}$ is assumed to be completed in $1 \mathrm{~s}$. The second is "mass-scaling", which is done by artificially increasing the density of elements to obtain a larger stable time-step.

Sometimes the effect of these strategies is insignificant and justifiable. This often occurs for cases where the mass adjustments is applied to just a few small elements in noncritical areas or quasi-static simulations where inertia effects are very small and the kinetic energy is negligible relative to the peak internal energy. In this study, the loading is quasi-static, but kink-banding is a dynamic event, which releases a large amount of kinetic energy, thus care has to be taken when adopting the above strategies.

\section{Interaction between Delamination and Kinking}

To determine the influence of delamination on kinking behavior and vice versa, a set of base values for the DCZM element properties that is typical of epoxy adhesives are assumed as shown in Table 4. The cohesive $\operatorname{strength}\left(\sigma_{c}\right.$ and $\left.\tau_{c}\right)$ values are then varied by multiplying the base values by a factor to determine the influence of changes in interfacial properties to the failure modes of the laminate. Also, the stress-strain response of an identical model, but with perfect interface bonding between the layers is used for comparison.

Table 4. Typical DCZM properties of epoxy adhesive

\begin{tabular}{cc}
\hline \hline$G_{I C}$ & $5 \frac{\mathrm{kJ}}{\mathrm{m}^{2}}$ \\
$G_{I I C}$ & $8.5 \frac{\mathrm{kJ}}{\mathrm{m}^{2}}$ \\
$\sigma_{C}$ & $9 \mathrm{MPa}$ \\
$\tau_{C}$ & $36 \mathrm{MPa}$ \\
\hline
\end{tabular}

From the computational results, it can be seen that with typical epoxy adhesive DCZM properties, the "DCZM", peak stress is barely affected as compared to the perfect interface case, suggesting that kink banding will prevail as the compression strength limiting mechanism for these properties. Not surprisingly, as DCZM properties are decreased, the peak stress decreases substantially. An interesting detail to note in Fig. 24, is that the load bearing capabilities of the "DCZM*10", "DCZM" and "DCZM/10" are very different, and this can be attributed to the effects of delamination. Fig. 26 clearly shows that with high 


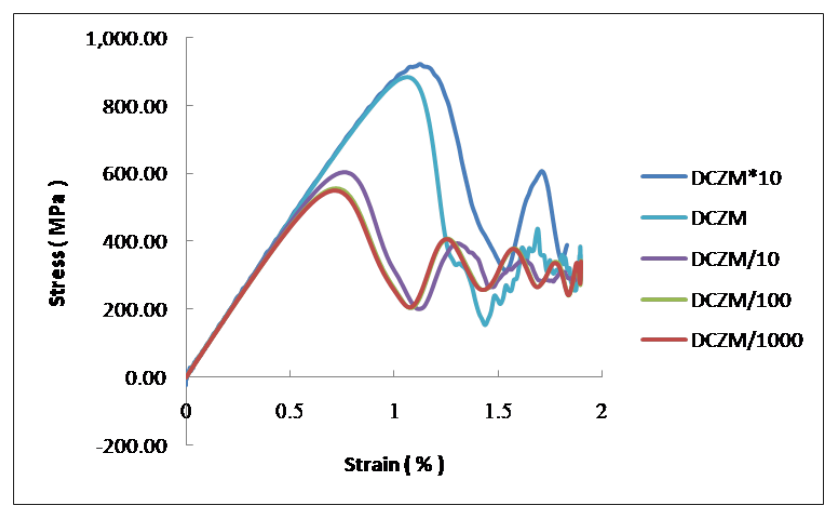

Figure 24. Global stress-strain results with varying DCZM properties

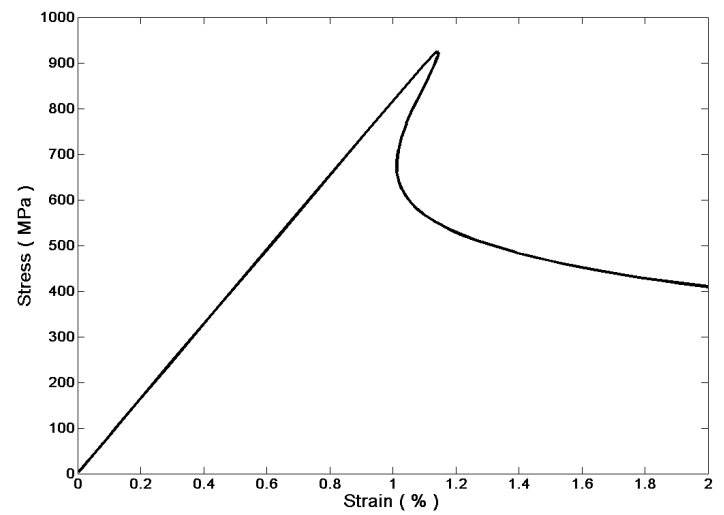

Figure 25. Global stress-strain results with perfect inter-laminar interface

DCZM properties, kink-banding can occur with no delamination in the post-peak regime. As the properties are lowered(Fig. 27), delamination starts to occur in the post-kinked regime. With even lower properties(Fig. 28), kink-banding is actually triggered due to delamination and delamination failure is seen to provide the compression strength limiting mechanism.

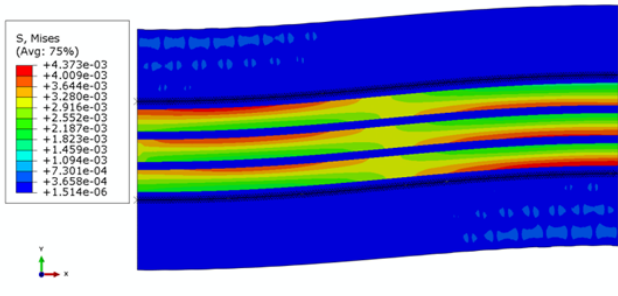

(a) Peak Load, Displacement $=1.40 \mu \mathrm{m}$

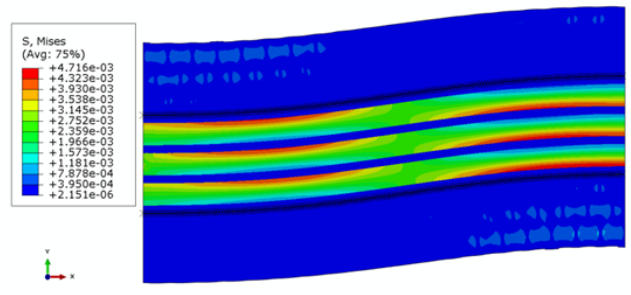

(b) Post-buckling, Displacement $=1.60 \mu \mathrm{m}$

Figure 26. "DCZM*10" : Buckling with no delamination

\section{Conclusions}

The experimental results show that, in Type A laminates, compressive strength is not affected by scaling of thickness of laminae, and failure is triggered by delamination at the interface of the outer layers. In Type C laminates, failure is triggered by kinking of the innermost layer. The computational results for the 8-layered 


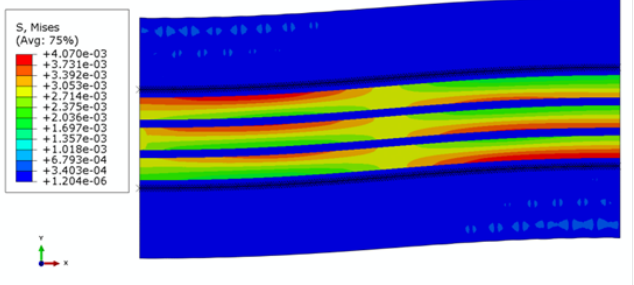

(a) Peak Load, Displacement $=1.38 \mu \mathrm{m}$

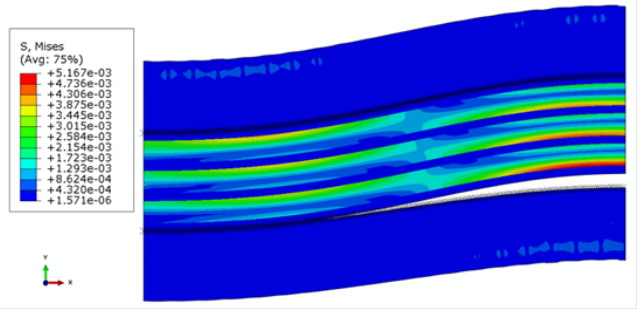

(b) Post-buckling, Displacement $=1.60 \mu \mathrm{m}$

Figure 27. "DCZM" : Buckling leading to delamination

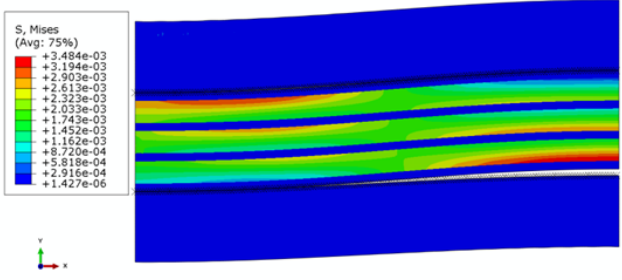

(a) Peak Load, Displacement $=0.925 \mu \mathrm{m}$

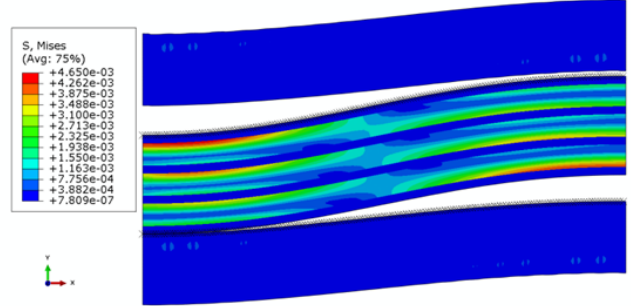

(b) Post-buckling, Displacement $=1.60 \mu \mathrm{m}$

Figure 28. "DCZM/10" : Delamination triggered buckling

model indicates that the initial stiffness lies in the range of the stiffnesses determined from experiments. The imperfection sensitivity analysis on the 8-layer model indicates that the peak stress reduces with increasing fiber misalignment, for a range of misalignment angles between 0.5-2 degrees.

Preliminary computational results with a 3-layer cross-ply model reveals the mechanics of interaction between kinking and delamination failure and how these modes are influenced by varying the interface fracture properties of the DCZM elements that are placed at the interface between the laminae. It is observed that as the interface properties are degraded, the load bearing capacity of the model reduces. Also, the mode of failure changes from kinking to delamination triggered kinking failure. Therefore, this model provides a pathway to capture failure mode interaction. Further refinements to the model and its adaptation to the 8-layer micro-laminate model is required in order to shed light on the differences observed in the experimental results of Type A and Type C laminates.

\section{Acknowledgments}

The authors are grateful for the financial sponsorship of this work through an AFOSR grant to Comet Technology Corporation, Inc., Ann Arbor and the University of Michigan, Ann Arbor.

\section{References}

${ }^{1}$ Ahn, J.H. and Waas, A.M., AIAA Journal, Vol.38, No. 12, Pages: 2299-2304, 2000.

${ }^{2}$ Ahn, J.H. and Waas, A.M., AIAA Journal, Vol.40, No. 2, Pages: 346-358, 2002.

${ }^{3}$ Schultheisz, C.R. and Waas, A.M., Progress in Aerospace Sciences, Vol.32, No.1, Pages: 1-42, 1996.

${ }^{4}$ Yerramalli, C.s. and Waas, A.M., IJSS, Vol.40, Pages: 11391164, 2003.

${ }^{5}$ Basu, S., Waas, A.M. and Ambur, D.R., JMPS, Vol.54, Pages: 611-634, 2006.

${ }^{6}$ Jumahat, A., Soutis, C., Jones, F.R. and Hodziv, A., Composite Structures, Vol.92, Pages: 295-305, 2010.

${ }^{7}$ Gustafson, P.A. and Waas, A.M., IJSS, Vol.46, No. 10, Pages: 2201-2215 , 2009.

${ }^{8}$ Yurgartis, S.W., IJSS, Vol.30, No. 4, Pages: 279-293, 1987.

${ }^{9}$ Basu, S., Waas, A.M. and Ambur, D.R., Journal of Mechanics of Materials and Structures, Vol. 1, No. 6, Pages: 979-1000, 2006 